\title{
Non-Markovian feature of the classical Hall effect
}

\author{
I.B. Abdurakhmanov ${ }^{1}$, G.G. Adamian², N.V. Antonenko², and Z. Kanokov ${ }^{2,3,4}$, \\ ${ }^{1}$ Curtin Institute for Computation, Department of Physics and Astronomy, \\ Curtin University, Perth, WA 6845, Australia \\ ${ }^{2}$ Joint Institute for Nuclear Research, 141980 Dubna, Russia \\ ${ }^{3}$ National University, 700174 Tashkent, Uzbekistan \\ ${ }^{4}$ Institute of Nuclear Physics, 702132 Tashkent, Uzbekistan
}

(Dated: June 19, 2021)

\begin{abstract}
The classical Hall effect resulting from the impact of external magnetic and electric fields on the non-Markovian dynamics of charge carriers is studied. The dependence of the tangent of the Hall angle on the magnetic field is derived and compared with the experimental data for Zn. The method is proposed to determine experimentally the memory time in a system.

PACS numbers: 09.37.-d, 03.40.-a, 03.65.-w, 24.60.-k

Keywords: classical Hall effect; cyclotron frequency; friction coefficients; Langevin formalism; nonMarkovian dynamics; electric field; magnetic field
\end{abstract}




\section{INTRODUCTION}

The behavior of solid matter under the influence of impact fields is one of the interesting

topics in solid-state physics [1 5]. The external field may be an electric field, a magnetic field, an optical signal, or a temperature gradient. Such external fields modify the distribution of internal energy which in turn modifies or alters the electronic properties, such as the carrier concentration or the carrier mobility. Besides the carrier mobility, the electric current is also affected by magnetic field which deflects its direction. Modeling the electric current implies the determination of the time-dependence of the number of electrons with the given momentum at certain location. The equations of motion for it can be obtained by using the quantum Langevin approach or density matrix formalism which is widely applied to find the effects of fluctuations and dissipation in macroscopic systems [6-16]. The Langevin method in the kinetic theory significantly simplifies the calculation of nonequilibrium quantum and thermal fluctuations and provides a clear picture of the dynamics of the process [14, 16-32].

The aim of the present work is the treatment of the classic Hall effect in the external constant magnetic and electric fields beyond the Markov approximation (instantaneus dissipation and delta-correlated fluctuations) and the weak-coupling limit. We generalize the Langevin formalism, which has been developed for non-Markovian noise in Refs. [18, 23], by including the external fields. The basic idea of our model is the following: we consider the center of mass of the charge carriers with the positive charge $e=|e|$ as a quantum particle coupled to the environment (heat bath) through particle-phonon interactions. In the solution of the second order Heisenberg equations for the heat bath degrees of freedom, the generalized non-Markovian Langevin equations of a quantum particle is directly obtained. At the same time, the memory function, which contains all the information concerning the effect of heat bath on the transport properties of the quantum particle, is also obtained without any approximations for the particle-phonon interaction.

The paper is organized as follows. In Sec. II, we give the Hamiltonian of the system and solve the generalized non-Markovian Langevin equations for a quantum particle. In Sec. III, we consider linear coupling in the coordinate between the heat bath and quantum particle which describes the center of mass of the charge carriers. The effects of heat-bath and external constant electric magnetic field on the dynamics of quantum particle are fully studied. The analytical expressions for macroscopically observable values are worked out in 
the context of the model. The classical Hall effect is considered. The cross component of electric field (which is absent at the initial time) generating by the magnetic field and heat bath is investigated at different system conditions. The developed model is used in Sec. IV to describe the experimental data on the classical Hall effect in Zn. The calculated results are shown to be in a good agreement with the experiment.

\section{NON-MARKOVIAN LANGEVIN EQUATIONS WITH EXTERNAL MAG- NETIC AND ELECTRIC FIELDS}

\section{A. Derivation of quantum Langevin equations}

Let us consider the two-dimensional motion of quantum charged particle in the presence of heat bath and external constant electric $\mathbf{E}=\left(E_{x}, 0,0\right)$ and magnetic fields $\mathbf{B}=(0,0, B)$. The total Hamiltonian of this system is

$$
H=H_{c}+H_{b}+H_{c b}
$$

The Hamiltonian $H_{c}$ describes the collective subsystem (quantum particle) with effective mass tensor and charge $e=|e|$ in electric and magnetic fields:

$$
H_{c}=\frac{1}{2 m_{x}}\left(p_{x}-e A_{x}(x, y)\right)^{2}+\frac{1}{2 m_{y}}\left(p_{y}-e A_{y}(x, y)\right)^{2}+e E_{x} x=\frac{\pi_{x}^{2}}{2 m_{x}}+\frac{\pi_{y}^{2}}{2 m_{y}}+e E_{x} x .
$$

Here, $m_{x}$ and $m_{y}$ are the components of the effective mass tensor, $\mathbf{R}=(x, y, 0)$ and $\mathbf{p}=$ $\left(p_{x}, p_{y}, 0\right)$ are the coordinate and canonically conjugated momentum, respectively, $\mathbf{A}=$ $\left(-\frac{1}{2} y B, \frac{1}{2} x B, 0\right)$ is the vector potential of the magnetic field, and the electric field $E_{x}$ acts in $x$ direction. For simplicity, in Eq. (2) we introduce the notations

$$
\pi_{x}=p_{x}+\frac{1}{2} m_{x} \omega_{c x} y, \quad \pi_{y}=p_{y}-\frac{1}{2} m_{y} \omega_{c y} x
$$

with frequencies $\omega_{c x}=\frac{e B}{m_{x}}$ and $\omega_{c y}=\frac{e B}{m_{y}}$. The cyclotron frequency is $\omega_{c}=\sqrt{\omega_{c x} \omega_{c y}}=\frac{e B}{\sqrt{m_{x} m_{y}}}$.

The second term in Eq. (1) represents the Hamiltonian of the phonon heat bath,

$$
H_{b}=\sum_{\nu} \hbar \omega_{\nu} b_{\nu}^{+} b_{\nu}
$$

where $b_{\nu}^{+}$and $b_{\nu}$ are the phonon creation and annihilation operators of the heat bath. The coupling between the heat bath and collective subsystem is described by

$$
H_{c b}=\sum_{\nu} V_{\nu}(\mathbf{R})\left(b_{\nu}^{+}+b_{\nu}\right)+\sum_{\nu} \frac{1}{\hbar \omega_{\nu}} V_{\nu}^{2}(\mathbf{R})
$$


The first term of Eq. (44) corresponds to the exchange of energy between the collective subsystem and heat bath. We introduce the counterterm (second term) in $H_{c b}$ in order to compensate the coupling-induced renormalization of the potential. Naturally, it can be always splitted off from $e E_{x} x$ in Eq. (2). In general case, $V_{\nu}(\mathbf{R})$ depends on a strength of magnetic field and the impact of $\mathbf{B}$ is entered into the dissipative kernels and random forces.

The equations of motion are

$$
\begin{aligned}
& \dot{x}(t)=\frac{i}{\hbar}[H, x]=\frac{\pi_{x}(t)}{m_{x}}, \quad \dot{y}(t)=\frac{i}{\hbar}[H, y]=\frac{\pi_{y}(t)}{m_{y}}, \\
& \dot{\pi}_{x}(t)=\frac{i}{\hbar}\left[H, \pi_{x}\right]=\pi_{y}(t) \omega_{c y}-e E_{x}-\sum_{\nu} V_{\nu, x}^{\prime}(\mathbf{R})\left(b_{\nu}^{+}+b_{\nu}\right)-2 \sum_{\nu} \frac{V_{\nu}(\mathbf{R}) V_{\nu, x}^{\prime}(\mathbf{R})}{\hbar \omega_{\nu}} \\
& \dot{\pi}_{y}(t)=\frac{i}{\hbar}\left[H, \pi_{y}\right]=-\pi_{x}(t) \omega_{c x}-\sum_{\nu} V_{\nu, y}^{\prime}(\mathbf{R})\left(b_{\nu}^{+}+b_{\nu}\right)-2 \sum_{\nu} \frac{V_{\nu}(\mathbf{R}) V_{\nu, y}^{\prime}(\mathbf{R})}{\hbar \omega_{\nu}}
\end{aligned}
$$

and

$$
\begin{aligned}
\dot{b}_{\nu}^{+}(t)=\frac{i}{\hbar}\left[H, b_{\nu}^{+}\right] & =i \omega_{\nu} b_{\nu}^{+}(t)+\frac{i}{\hbar} V_{\nu}(\mathbf{R}), \\
\dot{b}_{\nu}(t)=\frac{i}{\hbar}\left[H, b_{\nu}\right] & =-i \omega_{\nu} b_{\nu}(t)-\frac{i}{\hbar} V_{\nu}(\mathbf{R}) .
\end{aligned}
$$

The solution of Eqs. (6) are

$$
\begin{aligned}
& b_{\nu}^{+}(t)+b_{\nu}(t)=f_{\nu}^{+}(t)+f_{\nu}(t)-\frac{2 V_{\nu}(\mathbf{R})}{\hbar \omega_{\nu}}+\frac{2}{\hbar \omega_{\nu}} \int_{0}^{t} d \tau \dot{V}_{\nu}(\mathbf{R}(\tau)) \cos \left(\omega_{\nu}[t-\tau]\right), \\
& b_{\nu}^{+}(t)-b_{\nu}(t)=f_{\nu}^{+}(t)-f_{\nu}(t)+\frac{2 i}{\hbar \omega_{\nu}} \int_{0}^{t} d \tau \dot{V}_{\nu}(\mathbf{R}(\tau)) \sin \left(\omega_{\nu}[t-\tau]\right),
\end{aligned}
$$

where

$$
f_{\nu}(t)=\left[b_{\nu}(0)+\frac{1}{\hbar \omega_{\nu}} V_{\nu}(\mathbf{R}(0))\right] e^{-i \omega_{\nu} t} .
$$

Substituting (17) into (5) and eliminating the bath variables from the equations of motion for the collective subsystem, we obtain a set of nonlinear integro-differential stochastic dissipative equations

$$
\begin{aligned}
\dot{x}(t) & =\frac{\pi_{x}(t)}{m_{x}}, \quad \dot{y}(t)=\frac{\pi_{y}(t)}{m_{y}} \\
\dot{\pi}_{x}(t) & =\pi_{y}(t) \omega_{c y}-e E_{x}-\frac{1}{2} \int_{0}^{t} d \tau\left\{K_{x x}(t, \tau), \dot{x}(\tau)\right\}_{+}-\frac{1}{2} \int_{0}^{t} d \tau\left\{K_{x y}(t, \tau), \dot{x}(\tau)\right\}_{+}+F_{x}(t) \\
\dot{\pi}_{y}(t) & =-\pi_{x}(t) \omega_{c x}-\frac{1}{2} \int_{0}^{t} d \tau\left\{K_{y y}(t, \tau), \dot{y}(\tau)\right\}_{+}-\frac{1}{2} \int_{0}^{t} d \tau\left\{K_{y x}(t, \tau), \dot{y}(\tau)\right\}_{+}+F_{y}(t)
\end{aligned}
$$


The dissipative kernels and random forces in (8) are

$$
\begin{aligned}
& K_{x x}(t, \tau)=\sum_{\nu} \frac{1}{\hbar \omega_{\nu}}\left\{V_{\nu, x}^{\prime}(\mathbf{R}(t)), V_{\nu, x}^{\prime}(\mathbf{R}(\tau))\right\}_{+} \cos \left(\omega_{\nu}[t-\tau]\right), \\
& K_{x y}(t, \tau)=\sum_{\nu} \frac{1}{\hbar \omega_{\nu}}\left\{V_{\nu, x}^{\prime}(\mathbf{R}(t)), V_{\nu, y}^{\prime}(\mathbf{R}(\tau))\right\}_{+} \cos \left(\omega_{\nu}[t-\tau]\right), \\
& K_{y x}(t, \tau)=\sum_{\nu} \frac{1}{\hbar \omega_{\nu}}\left\{V_{\nu, y}^{\prime}(\mathbf{R}(t)), V_{\nu, x}^{\prime}(\mathbf{R}(\tau))\right\}_{+} \cos \left(\omega_{\nu}[t-\tau]\right), \\
& K_{y y}(t, \tau)=\sum_{\nu} \frac{1}{\hbar \omega_{\nu}}\left\{V_{\nu, y}^{\prime}(\mathbf{R}(t)), V_{\nu, y}^{\prime}(\mathbf{R}(\tau))\right\}_{+} \cos \left(\omega_{\nu}[t-\tau]\right)
\end{aligned}
$$

and

$$
\begin{aligned}
& F_{x}(t)=\sum_{\nu} F_{x}^{\nu}(t)=-\sum_{\nu} V_{\nu, x}^{\prime}(\mathbf{R}(t))\left[f_{\nu}^{+}(t)+f_{\nu}(t)\right], \\
& F_{y}(t)=\sum_{\nu} F_{y}^{\nu}(t)=-\sum_{\nu} V_{\nu, y}^{\prime}(\mathbf{R}(t))\left[f_{\nu}^{+}(t)+f_{\nu}(t)\right],
\end{aligned}
$$

respectively. Here, we use the notations: $V_{\nu, x}^{\prime}=\partial V_{\nu} / \partial x, V_{\nu, y}^{\prime}=\partial V_{\nu} / \partial y$, and $\left\{Z_{1}, Z_{2}\right\}_{+}=$ $Z_{1} Z_{2}+Z_{2} Z_{1}$. Following the usual procedure in statistical mechanics, we identify the operators $F_{x}^{\nu}$ and $F_{y}^{\nu}$ as fluctuations because of the uncertainty of the initial conditions for the bath operators. To specify the statistical properties of the fluctuations, we consider an ensemble of initial states in which the fluctuations have the Gaussian distribution with zero average value

$$
\ll F_{x}^{\nu}(t) \gg=\ll F_{y}^{\nu}(t) \gg=0
$$

Here, the symbol $\ll \ldots \gg$ denotes the average over the bath. Bose-Einstein statistics of the bath are

$$
\begin{aligned}
& \ll f_{\nu}^{+}(t) f_{\nu^{\prime}}^{+}\left(t^{\prime}\right) \gg=\ll f_{\nu}(t) f_{\nu^{\prime}}\left(t^{\prime}\right) \gg=0, \\
& \ll f_{\nu}^{+}(t) f_{\nu^{\prime}}\left(t^{\prime}\right) \gg=\delta_{\nu, \nu^{\prime}} n_{\nu} e^{i \omega_{\nu}\left[t-t^{\prime}\right]}, \\
& \ll f_{\nu}(t) f_{\nu^{\prime}}^{+}\left(t^{\prime}\right) \gg=\delta_{\nu, \nu^{\prime}}\left(n_{\nu}+1\right) e^{-i \omega_{\nu}\left[t-t^{\prime}\right]},
\end{aligned}
$$

where the occupation numbers $n_{\nu}=\left[\exp \left(\hbar \omega_{\nu} / T\right)-1\right]^{-1}$ for phonons depend on temperature $T$.

Using the properties (10) and (11) of random forces, we get the following symmetrized 
correlation functions $\varphi_{k k^{\prime}}^{\nu}\left(t, t^{\prime}\right)=\ll F_{k}^{\nu}(t) F_{k^{\prime}}^{\nu}\left(t^{\prime}\right)+F_{k^{\prime}}^{\nu}\left(t^{\prime}\right) F_{k}^{\nu}(t) \gg,\left(k, k^{\prime}=x, y\right)$ :

$$
\begin{aligned}
& \varphi_{x x}^{\nu}\left(t, t^{\prime}\right)=\left[2 n_{\nu}+1\right]\left\{V_{\nu, x}^{\prime}(\mathbf{R}(t)), V_{\nu, x}^{\prime}\left(\mathbf{R}\left(t^{\prime}\right)\right)\right\}_{+} \cos \left(\omega_{\nu}\left[t-t^{\prime}\right]\right), \\
& \varphi_{y y}^{\nu}\left(t, t^{\prime}\right)=\left.\varphi_{x x}^{\nu}\left(t, t^{\prime}\right)\right|_{x \rightarrow y}, \\
& \varphi_{x y}^{\nu}\left(t, t^{\prime}\right)=\left[2 n_{\nu}+1\right]\left\{V_{\nu, x}^{\prime}(\mathbf{R}(t)), V_{\nu, y}^{\prime}\left(\mathbf{R}\left(t^{\prime}\right)\right)\right\}_{+} \cos \left(\omega_{\nu}\left[t-t^{\prime}\right]\right), \\
& \varphi_{y x}^{\nu}\left(t, t^{\prime}\right)=\left.\varphi_{x y}^{\nu}\left(t, t^{\prime}\right)\right|_{x \rightarrow y} .
\end{aligned}
$$

The quantum fluctuation-dissipation relations read

$$
\begin{aligned}
& \sum_{\nu} \varphi_{x x}^{\nu}\left(t, t^{\prime}\right) \frac{\tanh \left[\frac{\hbar \omega_{\nu}}{2 T}\right]}{\hbar \omega_{\nu}}=K_{x x}\left(t, t^{\prime}\right), \\
& \sum_{\nu} \varphi_{y y}^{\nu}\left(t, t^{\prime}\right) \frac{\tanh \left[\frac{\hbar \omega_{\nu}}{2 T}\right]}{\hbar \omega_{\nu}}=K_{y y}\left(t, t^{\prime}\right), \\
& \sum_{\nu} \varphi_{x y}^{\nu}\left(t, t^{\prime}\right) \frac{\tanh \left[\frac{\hbar \omega_{\nu}}{2 T}\right]}{\hbar \omega_{\nu}}=K_{x y}\left(t, t^{\prime}\right), \\
& \sum_{\nu} \varphi_{y x}^{\nu}\left(t, t^{\prime}\right) \frac{\tanh \left[\frac{\hbar \omega_{\nu}}{2 T}\right]}{\hbar \omega_{\nu}}=K_{y x}\left(t, t^{\prime}\right) .
\end{aligned}
$$

The validity of the fluctuation-dissipation relations means that we have properly identified the dissipative terms in the non-Markovian dynamical equations of motion. The quantum fluctuation-dissipation relations differ from the classical ones and are reduced to them in the limit of high temperature.

\section{B. Derivation of non-stationary transport coefficients}

In order to solve the equations of motion (8) for the collective variables, we applied the Laplace transformation. It significantly simplifies the solution of the problem. After the Laplace transformation, the equations of motion take as

$$
\begin{gathered}
x(s) s=x(0)+\frac{\pi_{x}(s)}{m_{x}}, \quad y(s) s=y(0)+\frac{\pi_{y}(s)}{m_{y}}, \\
\pi_{x}(s) s+\frac{\pi_{x}(s)}{m_{x}}\left(K_{x x}(s)+K_{x y}(s)\right)=\pi_{x}(0)+\omega_{c y} \pi_{y}(s)-\frac{1}{s} e E_{x}+F_{x}(s), \\
\pi_{y}(s) s+\frac{\pi_{y}(s)}{m_{y}}\left(K_{y y}(s)+K_{y x}(w)\right)=\pi_{y}(0)-\omega_{c x} \pi_{x}(s)+F_{y}(s) .
\end{gathered}
$$

Here, $K_{x x}(s), K_{y y}(s), K_{x y}(s), K_{y x}(s)$ and $F_{x}(s), F_{y}(s)$ are the Laplace transforms of the dissipative kernels and random forces, respectively. For the solution of this system of equations, 
one should find the roots of the determinant

$$
D=s\left(m_{x} m_{y} \omega_{c}^{2}+\left[K_{x x}(s)+K_{x y}(s)+m_{x} s\right]\left[K_{y y}(s)+K_{y x}(s)+m_{y} s\right]\right)=0 .
$$

The explicit solutions for the originals are

$$
\begin{aligned}
x(t) & =x(0)+A_{1}(t) \pi_{x}(0)+A_{2}(t) \pi_{y}(0)-A_{3}(t) e E_{x}+I_{x}(t)+I_{x}^{\prime}(t), \\
y(t) & =y(0)+B_{1}(t) \pi_{y}(0)-B_{2}(t) \pi_{x}(0)+B_{3}(t) e E_{x}-I_{y}(t)+I_{y}^{\prime}(t), \\
\pi_{x}(t) & =C_{1}(t) \pi_{x}(0)+C_{2}(t) \pi_{y}(0)-C_{3}(t) e E_{x}+I_{\pi_{x}}(t)+I_{\pi_{x}}^{\prime}(t), \\
\pi_{y}(t) & =D_{1}(t) \pi_{y}(0)-D_{2}(t) \pi_{x}(0)+D_{3}(t) e E_{x}-I_{\pi_{y}}(t)+I_{\pi_{y}}^{\prime}(t),
\end{aligned}
$$

where

$$
\begin{aligned}
I_{x}(t)=\int_{0}^{t} A_{1}(\tau) F_{x}(t-\tau) d \tau, & I_{x}^{\prime}(t)=\int_{0}^{t} A_{2}(\tau) F_{y}(t-\tau) d \tau, \\
I_{y}(t)=\int_{0}^{t} B_{2}(\tau) F_{x}(t-\tau) d \tau, & I_{y}^{\prime}(t)=\int_{0}^{t} B_{1}(\tau) F_{y}(t-\tau) d \tau, \\
I_{\pi_{x}}(t)=\int_{0}^{t} C_{1}(\tau) F_{x}(t-\tau) d \tau, & I_{\pi_{x}}^{\prime}(t)=\int_{0}^{t} C_{2}(\tau) F_{y}(t-\tau) d \tau, \\
I_{\pi_{y}}(t)=\int_{0}^{t} D_{2}(\tau) F_{x}(t-\tau) d \tau, & I_{\pi_{y}}^{\prime}(t)=\int_{0}^{t} D_{1}(\tau) F_{y}(t-\tau) d \tau
\end{aligned}
$$

and the following time-dependent coefficients:

$$
\begin{aligned}
& A_{1}(t)=\hat{L}^{-1}\left[\frac{K_{y y}(s)+K_{y x}(s)+m_{y} s}{D}\right]=\left.B_{1}(t)\right|_{x \leftrightarrow y}, \\
& A_{2}(t)=m_{y} \omega_{c y} \hat{L}^{-1}\left[\frac{1}{D}\right]=\left.B_{2}(t)\right|_{x \leftrightarrow y}, \\
& A_{3}(t)=\hat{L}^{-1}\left[\frac{K_{y y}(s)+K_{y x}(s)+m_{y} s}{s D}\right], \quad B_{3}(t)=m_{x} \omega_{c x} \hat{L}^{-1}\left[\frac{1}{s D}\right], \\
& C_{1}(t)=m_{x} \hat{L}^{-1}\left[\frac{s\left(K_{y y}(s)+K_{y x}(s)+m_{y} s\right)}{D}\right]=\left.D_{1}(t)\right|_{x \leftrightarrow y}, \\
& C_{2}(t)=m_{x} m_{y} \omega_{c y} \hat{L}^{-1}\left[\frac{s}{D}\right]=\left.D_{2}(t)\right|_{x \leftrightarrow y}, \\
& C_{3}(t)=m_{x} \hat{L}^{-1}\left[\frac{K_{y y}(s)+K_{y x}(s)+m_{y} s}{D}\right], \quad D_{3}(t)=m_{x} m_{y} \omega_{c x} \hat{L}^{-1}\left[\frac{1}{D}\right] .
\end{aligned}
$$

Here, $\hat{L}^{-1}$ denotes the inverse Laplace transformation. The exact solutions of $x(t), y(t)$, $\pi_{x}(t)$, and $\pi_{y}(t)$ in terms of roots $s_{i}$ are given by the residue theorem.

In order to determine the transport coefficients, we use Eqs. (17). Averaging them over the whole system and by differentiating in $t$, we obtain the system of equations for the first 
moments:

$$
\begin{aligned}
<\dot{x}(t)> & =\frac{<\pi_{x}(t)>}{m_{x}}, \quad<\dot{y}(t)>=\frac{<\pi_{y}(t)>}{m_{y}} \\
<\dot{\pi}_{x}(t)> & =\tilde{\omega}_{c y}(t)<\pi_{y}(t)>-\lambda_{\pi_{x}}(t)<\pi_{x}(t)>-e \tilde{E}_{x x}(t) \\
<\dot{\pi}_{y}(t)> & =-\tilde{\omega}_{c x}(t)<\pi_{x}(t)>-\lambda_{\pi_{y}}(t)<\pi_{y}(t)>-e \tilde{E}_{x y}(t),
\end{aligned}
$$

where the friction coefficients are

$$
\begin{aligned}
& \lambda_{\pi_{x}}(t)=-\frac{D_{1}(t) \dot{C}_{1}(t)+D_{2}(t) \dot{C}_{2}(t)}{C_{1}(t) D_{1}(t)+C_{2}(t) D_{2}(t)} \\
& \lambda_{\pi_{y}}(t)=-\frac{C_{1}(t) \dot{D}_{1}(t)+C_{2}(t) \dot{D}_{2}(t)}{C_{1}(t) D_{1}(t)+C_{2}(t) D_{2}(t)}
\end{aligned}
$$

and the renormalized cyclotron frequencies are given by

$$
\begin{gathered}
\tilde{\omega}_{c x}(t)=\frac{D_{1}(t) \dot{D}_{2}(t)-D_{2}(t) \dot{D}_{1}(t)}{C_{1}(t) D_{1}(t)+C_{2}(t) D_{2}(t)}, \\
\tilde{\omega}_{c y}(t)=\frac{C_{1}(t) \dot{C}_{2}(t)-C_{2}(t) \dot{C}_{1}(t)}{C_{1}(t) D_{1}(t)+C_{2}(t) D_{2}(t)},
\end{gathered}
$$

while the components of electric field read:

$$
\begin{aligned}
& \tilde{E}_{x x}(t)=E_{x}\left[D_{3}(t) \tilde{\omega}_{c y}(t)+C_{3}(t) \lambda_{\pi_{x}}(t)+\dot{C}_{3}(t)\right] \\
& \tilde{E}_{x y}(t)=E_{x}\left[C_{3}(t) \tilde{\omega}_{c x}(t)-D_{3}(t) \lambda_{\pi_{y}}(t)-\dot{D}_{3}(t)\right]
\end{aligned}
$$

As seen, the dynamics is governed by the non-stationary coefficients. It should be noted that the cross component $\tilde{E}_{x y}(t)$ of electric field is absent at the initial time and appears during the evolution of system.

\section{LINEAR COUPLING IN COORDINATE WITH HEAT BATH}

\section{A. Solution of Non-Markovian Langevin equations}

For the system with linear coupling in coordinate, the coupling term is written as

$$
H_{c b}=\sum_{\nu}\left(\alpha_{\nu} x+\beta_{\nu} y\right)\left(b_{\nu}^{+}+b_{\nu}\right)+\sum_{\nu} \frac{1}{\hbar \omega_{\nu}}\left(\alpha_{\nu} x+\beta_{\nu} y\right)^{2},
$$

where $\alpha_{\nu}$ and $\beta_{\nu}$ are real coupling constants. Here, we again introduce the counter term which depends on the coordinates of the collective system and can be treated as a part of 
the potential. The operators of random forces and dissipative kernels in Eqs. (8) are

$$
F_{x}(t)=-\sum_{\nu} \alpha_{\nu}\left(f_{\nu}^{+}+f_{\nu}\right), \quad F_{y}(t)=-\sum_{\nu} \beta_{\nu}\left(f_{\nu}^{+}+f_{\nu}\right)
$$

and

$$
\begin{aligned}
& K_{x x}(t-\tau)=\sum_{\nu} \frac{2 \alpha_{\nu}^{2}}{\hbar \omega_{\nu}} \cos \left(\omega_{\nu}[t-\tau]\right), \\
& K_{y y}(t-\tau)=\sum_{\nu} \frac{2 \beta_{\nu}^{2}}{\hbar \omega_{\nu}} \cos \left(\omega_{\nu}[t-\tau]\right),
\end{aligned}
$$

respectively. Here, we assume that there are no correlations between $F_{x}^{\nu}$ and $F_{y}^{\nu}$, so that $K_{x y}=K_{y x}=0$. If the coupling constants $\alpha_{\nu}$ and $\beta_{\nu}$ depend on magnetic field, then the dissipative kernels $K_{x x}$ and $K_{y y}$ are the functions of $B$.

It is convenient to introduce the spectral density $D_{\omega}$ of the heat bath excitations to replace the sum over different oscillators, $\nu$, by an integral over the frequency: $\sum_{\nu} \ldots \rightarrow \int_{0}^{\infty} d \omega D_{\omega} \ldots$ This replacement is accompanied by the following replacements: $\alpha_{\nu} \rightarrow \alpha_{\omega}, \beta_{\nu} \rightarrow \beta_{\omega}^{0}, \omega_{\nu} \rightarrow \omega$, and $n_{\nu} \rightarrow n_{\omega}$. Let us consider the following spectral functions [14]

$$
D_{\omega} \frac{\left|\alpha_{\omega}\right|^{2}}{\hbar \omega}=\frac{\alpha^{2}}{\pi} \frac{\gamma^{2}}{\gamma^{2}+\omega^{2}}, \quad D_{\omega} \frac{\left|\beta_{\omega}\right|^{2}}{\hbar \omega}=\frac{\beta^{2}}{\pi} \frac{\gamma^{2}}{\gamma^{2}+\omega^{2}}
$$

where the memory time $\gamma^{-1}$ of the dissipation is inverse to the phonon bandwidth of the heat bath excitations which are coupled to a quantum particle. This is the Ohmic dissipation with the Lorentian cutoff (Drude dissipation) [6 -11, 14, 23].

Using the spectral functions (25), we obtain the dissipative kernels and their Laplace transforms in convenient forms

$$
\begin{aligned}
K_{x x}(t) & =m_{x} \lambda_{x} \gamma e^{-\gamma|t|}, & K_{y y}(t) & =m_{y} \lambda_{y} \gamma e^{-\gamma|t|}, \\
K_{x x}(s) & =\frac{m_{x} \lambda_{x} \gamma}{s+\gamma}, & K_{y y}(s) & =\frac{m_{y} \lambda_{y} \gamma}{s+\gamma},
\end{aligned}
$$

where the coefficients

$$
\lambda_{x}=\hbar \alpha^{2}=\frac{1}{m_{x}} \int_{0}^{\infty} K_{x x}(t-\tau) d \tau, \quad \lambda_{y}=\hbar \beta^{2}=\frac{1}{m_{y}} \int_{0}^{\infty} K_{y y}(t-\tau) d \tau
$$

are the friction coefficients in Markovian limit. So, the solutions for the collective variables 
(17) include the following time-dependent coefficients:

$$
\begin{aligned}
A_{1}(t) & =\dot{A}_{3}(t), \quad A_{2}(t)=\left.\dot{B}_{3}(t)\right|_{x \leftrightarrow y}, \\
A_{3}(t) & =\frac{1}{m_{x}}\left(\frac{\lambda_{y}}{\lambda_{x} \lambda_{y}+\omega_{c}^{2}} t+\frac{\omega_{c}^{2}\left(\gamma-\lambda_{y}\right)-\lambda_{y}^{2}\left(\gamma-\lambda_{x}\right)}{\gamma\left(\lambda_{x} \lambda_{y}+\omega_{c x} \omega_{c y}\right)^{2}}\right. \\
& \left.+\sum_{i=1}^{4} \frac{b_{i} e^{s_{i} t}\left(\gamma+s_{i}\right)\left(\gamma \lambda_{y}+s_{i}\left(\gamma+s_{i}\right)\right)}{s_{i}^{2}}\right), \\
B_{1}(t) & =\left.\dot{A}_{3}(t)\right|_{x \leftrightarrow y}, \quad B_{2}(t)=\dot{B}_{3}(t), \\
B_{3}(t) & =\frac{\omega_{c x}}{m_{y}}\left(\frac{t}{\lambda_{x} \lambda_{y}+\omega_{c x} \omega_{c y}}+\frac{2 \lambda_{x} \lambda_{y}-\gamma\left(\lambda_{x}+\lambda_{y}\right)}{\gamma\left(\lambda_{x} \lambda_{y}+\omega_{c x} \omega_{c y}\right)^{2}}+\sum_{i=1}^{4} \frac{b_{i} e^{s_{i} t}\left(\gamma+s_{i}\right)^{2}}{s_{i}^{2}}\right), \\
C_{1}(t) & =m_{x} \ddot{A_{3}}(t), \quad C_{2}(t)=m_{x} \ddot{B}_{3}(t), \quad C_{3}(t)=m_{x} \dot{A}_{3}(t), \\
D_{1}(t) & =\left.C_{1}(t)\right|_{x \leftrightarrow y}, \quad D_{2}(t)=m_{y} \ddot{B}_{3}(t), \quad D_{3}(t)=m_{y} \dot{B}_{3}(t),
\end{aligned}
$$

where $b_{i}=\left[\prod_{j \neq i}\left(s_{i}-s_{j}\right)\right]^{-1}$ with $i, j=1,2,3,4$ and $s_{i}$ are the roots of the equation

$$
\gamma \lambda_{x}\left[\gamma \lambda_{y}+s(\gamma+s)\right]+(\gamma+s)\left(s\left[s^{2}+\omega_{c}^{2}\right]+\gamma\left[\omega_{c}^{2}+s\left(\lambda_{y}+s\right)\right]\right)=0
$$

\section{B. Asymptotic friction coefficients, renormalized cyclotron frequency, and com-} ponents of the electric field

Using the relationship $s_{1} s_{2} s_{3} s_{4}=\gamma^{2}\left(\lambda_{x} \lambda_{y}+\omega_{c}^{2}\right)$ between the roots of Eq. (28), we obtain the asymptotic $(t \rightarrow \infty)$ expressions for the friction coefficients

$$
\begin{aligned}
\lambda_{\pi_{x}}(\infty) & =-\frac{\left[\gamma+s_{1}+s_{2}\right]\left[\gamma \lambda_{x}+\omega_{c}^{2}+\left(s_{1}+\gamma\right)\left(s_{1}+s_{2}\right)+s_{2}^{2}\right]}{\left(\gamma+s_{1}+s_{2}\right)^{2}+\omega_{c}^{2}} \\
\lambda_{\pi_{y}}(\infty) & =-\frac{\left[\gamma+s_{1}+s_{2}\right]\left[\gamma \lambda_{y}+\omega_{c}^{2}+\left(s_{1}+\gamma\right)\left(s_{1}+s_{2}\right)+s_{2}^{2}\right]}{\left(\gamma+s_{1}+s_{2}\right)^{2}+\omega_{c}^{2}}
\end{aligned}
$$

renormalized frequencies

$$
\begin{aligned}
\tilde{\omega}_{c x}(\infty) & =\frac{\omega_{c x}\left[\left(s_{1}+\gamma\right)\left(s_{2}+\gamma\right)-\gamma \lambda_{x}\right]}{\left(\gamma+s_{1}+s_{2}\right)^{2}+\omega_{c}^{2}} \\
\tilde{\omega}_{c y}(\infty) & =\frac{\omega_{c y}\left[\left(s_{1}+\gamma\right)\left(s_{2}+\gamma\right)-\gamma \lambda_{y}\right]}{\left(\gamma+s_{1}+s_{2}\right)^{2}+\omega_{c}^{2}}
\end{aligned}
$$

renormalized cyclotron frequency

$$
\tilde{\omega}_{c}(\infty)=\omega_{c} \frac{\left[\left(s_{1}+\gamma\right)\left(s_{2}+\gamma\right)-\gamma \lambda_{x}\right]^{\frac{1}{2}}\left[\left(s_{1}+\gamma\right)\left(s_{2}+\gamma\right)-\gamma \lambda_{y}\right]^{\frac{1}{2}}}{\left(\gamma+s_{1}+s_{2}\right)^{2}+\omega_{c}^{2}}=\sqrt{\tilde{\omega}_{c x} \tilde{\omega}_{c y}}
$$


and components of the electric field

$$
\begin{aligned}
& \tilde{E}_{x x}(\infty)=\frac{E_{x}}{\lambda_{x} \lambda_{y}+\omega_{c}^{2}}\left[\omega_{c x} \tilde{\omega}_{c y}(\infty)+\lambda_{y} \lambda_{\pi_{x}}(\infty)\right], \\
& \tilde{E}_{x y}(\infty)=\frac{E_{x}}{\lambda_{x} \lambda_{y}+\omega_{c}^{2}}\left[\lambda_{y} \tilde{\omega}_{c x}(\infty)-\omega_{c x} \lambda_{\pi_{y}}(\infty)\right],
\end{aligned}
$$

where $s_{1}$ and $s_{2}$ are the roots with the smallest absolute values of their real parts. In the case of zero external magnetic field $(B=0)$, or Markovian limit $(\gamma \rightarrow \infty)$, the cross current

disappears because of $\tilde{E}_{x y}(\infty)=0$. Moreover it also disappears at $\lambda_{y}=0$. Thus, if the particle can move freely in the cross direction, i.e. its time-of-flight in this direction is $\tau_{y} \sim 1 / \lambda_{y}=\infty$, there is no cross electric field $\left(E_{x y}=0\right)$. This important result follows from Eqs. (29)-(32). So, in the superconductive materials the Hall phenomenon should not be observed.

\section{Axial symmetric system}

One can obtain clearer physical picture of the process, if the space-symmetric system is considered. In this system $m_{x}=m_{y}=m, \lambda_{x}=\lambda_{y}=\lambda$, and $\omega_{c x}=\omega_{c y}=\omega_{c}$. So, the equation (28), which defines the poles in the integrands of $I_{j}$ and $I_{j}^{\prime}\left(j=x, y, \pi_{x}, \pi_{y}\right)$, is simplified:

$$
\left(s^{2}+\omega_{c}^{2}\right)(\gamma+s)^{2}+2 \gamma \lambda s(\gamma+s)+\lambda^{2} \gamma^{2}=0
$$

This equation has analytic roots:

$$
\begin{array}{lll}
s_{1}=-\frac{1}{2}\left(\gamma+i \omega_{c}+\sqrt{\left(\gamma-i \omega_{c}\right)^{2}-4 \gamma \lambda}\right), & s_{2}=s_{1}^{*}, \\
s_{3}=-\frac{1}{2}\left(\gamma+i \omega_{c}-\sqrt{\left(\gamma-i \omega_{c}\right)^{2}-4 \gamma \lambda}\right), & s_{4}=s_{3}^{*} .
\end{array}
$$

In order to split the real and imaginary parts of the roots, we expand them up to the first order in $\lambda / \gamma$ :

$$
\begin{aligned}
& s_{1}=-\frac{\lambda \gamma^{2}}{\gamma^{2}+\omega_{c}^{2}}-i \frac{\omega_{c}^{2}+\gamma^{2}+\lambda \gamma}{\gamma^{2}+\omega_{c}^{2}} \omega_{c} \\
& s_{3}=-\gamma \frac{\gamma^{2}+\omega_{c}^{2}-\gamma \lambda}{\gamma^{2}+\omega_{c}^{2}}+i \frac{\lambda \gamma \omega_{c}}{\gamma^{2}+\omega_{c}^{2}} .
\end{aligned}
$$

Using the expansion of

$$
\begin{aligned}
& \tilde{\omega}_{c}=\tilde{\omega}_{c}(\infty)=\frac{\omega_{c}}{2}-\frac{i}{4}\left(\sqrt{\left(\gamma-i \omega_{c}\right)^{2}-4 \gamma \lambda}+\sqrt{\left(\gamma+i \omega_{c}\right)^{2}-4 \gamma \lambda}\right) \\
& \lambda_{\pi}=\lambda_{\pi_{x}}(\infty)=\lambda_{\pi_{y}}(\infty)=\frac{\gamma}{2}+\frac{i}{4}\left(\sqrt{\left(\gamma-i \omega_{c}\right)^{2}-4 \gamma \lambda}-\sqrt{\left(\gamma+i \omega_{c}\right)^{2}-4 \gamma \lambda}\right)
\end{aligned}
$$


up to the first order in $\lambda / \gamma$

$$
\begin{aligned}
\tilde{\omega}_{c} & =\omega_{c}\left(1+\lambda_{\pi} / \gamma\right), \\
\lambda_{\pi} & =\frac{\gamma^{2}}{\gamma^{2}+\omega_{c}^{2}} \lambda
\end{aligned}
$$

we obtain from Eq. (32) the analytical expressions for the components of electric field:

$$
\begin{aligned}
& \frac{\tilde{E}_{x x}(\infty)}{E_{x}}=1+\frac{\lambda_{\pi}}{\gamma} \\
& \frac{\tilde{E}_{x y}(\infty)}{E_{x}}==\frac{\omega_{c} \lambda}{\gamma^{2}+\omega_{c}^{2}}=\frac{\omega_{c} \lambda_{\pi}}{\gamma^{2}}=\frac{\tilde{\omega}_{c}-\omega_{c}}{\gamma} .
\end{aligned}
$$

As seen, $\tilde{E}_{x y}(\infty) \rightarrow 0$ at $\gamma \rightarrow \infty$ or at $\lambda \rightarrow 0$, or $\omega_{c} \rightarrow 0$. From the expression for the nondiagonal component $\tilde{E}_{x y}(\infty) / E_{x}$ of electric field, we can find the magnitude of magnetic field when it reaches the maximum:

$$
\omega_{c}^{\max } \approx \gamma
$$

The formula (37) may serve as the simplest way of definition of the memory time $\gamma^{-1}$ of the dissipation in the system. Thus, in order to determine $\gamma$ of any system, it is necessary to determine the magnetic field at which the resulting cross electric field $\tilde{E}_{x y}(\infty)$ reaches its maximum value.

\section{CALCULATED RESULTS AND DISCUSSIONS}

In the model considered, one can study friction, cyclotron frequency, and external parameters of the system: the longitudal and cross components of electrical field. In the calculations we set $\lambda=\lambda_{x}=\lambda_{y}\left(\right.$ or $\left.\lambda_{\pi}=\lambda_{\pi_{x}}=\lambda_{\pi_{y}}\right)$ and $m=m_{x}=m_{y}\left(\right.$ or $\left.\omega_{c}=\omega_{c x}=\omega_{c y}\right)$.

\section{A. Friction coefficient, renormalized cyclotron frequency, and electrical field}

The dependencies of $\lambda_{\pi}, \tilde{\omega}_{c}, \tilde{E}_{x x}$, and $\tilde{E}_{x y}$ on time are shown in Figs. 1 and 2. The nonMarkovian correction to the friction coefficient increases with asymptotic friction coefficient (right side) and decreases with the magnetic field (left side). The increasing friction and magnetic field contribute to the rise of asymptotical microscopic magnetic field (bottom parts of Fig. 1). One can see in Fig. 2 that the cross electric field increases with the magnetic 
field, while the correction to the longitudinal electric field decreases. In general, the rise of the asymptotic friction coefficient increases the transient time of $\lambda_{\pi}, \tilde{\omega}_{c}, \tilde{E}_{x x}$, and $\tilde{E}_{x y}$. The change rate of the cyclotron frequency is about $\left(\tilde{\omega}_{c}-\omega_{c}\right) \gamma / 2$. One should reveal the reason of an increase of the energy of cyclotron rotation. The magnetic forces are perpendicular to the velocity of charge carrier and do not affect the energy. The dissipation and external magnetic field affect each other due to the non-Markovian dynamics of quantum system and the value of magnetic field is changed.

The asymptotic behaviors of the transport coefficients considered above are shown in Figs. 3 and 4. The effective friction coefficient decreases with increasing value of $\omega_{c}$ (Fig. 3). Note that the resistance obtained in our model does not depend on the magnetic field because we neglect the influence of magnetic field on the coupling between the quantum particle and heat-bath (or on the dissipative kernels). Moreover the curves corresponding to larger $\gamma$ have a weaker decreasing tendency and the friction does not depend on magnetic field in the Markovian limit, $\gamma \rightarrow \infty$. In the plot showing the dependence of effective friction $\lambda_{\pi}$ on the Markovian friction $\lambda$, the line is inclined less then 45 degrees to the abscissa. This means the friction coefficient has relatively small influence the system excepting the cases of very weak magnetic fields.

Analyzing the dependence of the frequency of microscopic magnetic field $\tilde{\omega}_{c}$ (Fig. 3) and effective electric field $\tilde{E}_{x x}$ (Fig. 4) on $\lambda$, one can conclude that the specimen with nonzero friction is more susceptible to the external magnetic and electrical fields. The non-Markovian corrections to the external magnetic and electrical fields are larger for the system with larger time of response $\gamma^{-1}$. In the upper parts of Fig. 4, the behavior of the cross electrical field is demonstrated. Relating the plots in Fig. 4, the dependence of the tangent of the Hall angle $\tilde{E}_{x y}(\infty) / \tilde{E}_{x x}(\infty)$ on friction and frequency of external magnetic field are obtained, and the key conclusions might be made. Firstly, the classical Hall phenomenon could not be observed at zero magnetic field or in the systems with zero friction coefficient. A number of experiments with superconductors [33] supports this conclusion. Secondly, according to the curves given in Fig. 4, the tangent of the Hall angle reaches its maximum at the strength $\omega_{c} \approx \gamma$ of magnetic field. Thirdly, Eqs. (35) and our numerical calculations suggest that the cross electric field is not originated in the Markovian limit $(\gamma \rightarrow \infty)$. Thus, taking non-Markovian nature of the system into account, one can explain the Hall phenomenon. 


\section{B. Application of model to interpretation of Hall angle experiment}

To demonstrate the possibilities of the model, we calculate the tangent of the Hall angle, $\tan \left[\Theta_{H}\right]=\tilde{E}_{x y}(\infty) / \tilde{E}_{x x}(\infty)$, for the sample of Zn settled in the increasing external magnetic field at two temperatures. Many experiments were performed to measure this value in several elements. We choose $\mathrm{Zn}$ because it has one type of charge carriers [34] and consequently easy to understand the technique of implementation of the model. In the case of matters with more then one type of charge carriers the problem is more complicated, since the twoband model given above should be considered. In order to turn to the observable values in expressions (32), all parameters in these expressions should be multiplied by the mass to charge ratio $m / e$. As a result, instead of the friction coefficient $\lambda$, cyclotron frequency $\omega_{c}$, and inverse response time $\gamma$ of the system we have the inverse reciprocal mobility of charge carriers $1 / \mu$, intensity of the magnetic field $B$ and new parameter $\Gamma=m \gamma / e$. From the experimental data [34] we may define the strength of the magnetic field at which the charge carriers deviate to the maximal angle from their non-field direction. Knowing the field, we define the parameter $\Gamma$ by using expression (37). Calculated and experimental characteristics of $\mathrm{Zn}$ are given in Table I. The calculations performed with values of mobility given in the table are in a good agreement with the experimental data (Fig. 5), specially at high strength of magnetic field.

\section{SUMMARY}

The behavior of the generated flow of charge carriers under the influence of external magnetic and electric fields was investigated in the two-dimensional case using the nonMarkovian Langevin approach and the general coupling between charge carriers and environment. The developed model was applied to the case where the collective variables are linear coupled in the coordinate with the variables of the heat-bath. In order to average the influence of heat-bath on the collective system, we applied the spectral function of heatbath excitations which describes Drude dissipation with Lorenzian cutoffs. The classical Hall effect was considered. We showed that the cross electric field (which is absent at the initial time) does not appear in the Markovian limit. So, taking non-Markovian nature of

the system into consideration, one can explain the classical Hall effect. The dependence of 
the tangent of the Hall angle on the magnetic field was investigated. Its value increases up to a specific magnitude and then monotonically decreases. The position of the maximum is

defined by the memory time $\gamma^{-1}$. One can suggest the method for determining memory time by measuring the magnetic field at which the resulting cross electric field has a maximum value. The possibilities of the model were shown through its application to interpret the experiment for the Zn sample. The calculated results agree well with the experimental data.

\section{Acknowledgments}

This work was partially supported by the Russian Foundation for Basic Research (Moscow) and DFG (Bonn). The IN2P3(France)-JINR(Dubna) Cooperation Programme is gratefully acknowledged.

[1] S.V. Vonsowsky, Magnetism (Nauka Publishers, Moscow, 1971).

[2] Ch. Kittel, Quantum Theory of Solids (John Wiley \& Sons, Inc., USA, 1987).

[3] V.V. Dodonov and V.I. Man'ko, Sov.J.Theor.Math.Phys., 65, 1 (1985).

[4] L. Langer et al., Phys. Rev. Lett. 76, 479 (1996); L. Jacak, P. Hawrylak, and A. Wojs, Quantum Dots (Springer-Verlag, Berlin, Heidelberg, New York, 1997).

[5] W.D. Heiss and R.G. Nazmitdinov, Phys.Rev. B 55, 16310 (1997). Pis'ma v ZhETF 68, 870 (1998); M. Dineykhan and R.G. Nazmitdinov, Phys. Rev. B 55, 13707 (1997); R.G. Nazmitdinov, N.S. Simonovic and J.M. Rost, Phys.Rev. B 65, 155307 (2002); Yu. Demidenko, A. Kuzyk, V. Lozovski, and O. Tretyak, J. Phys. C 16, 543 (2004); A. Matulis and E. Anisimovas, J. Phys. C 17, 3851 (2005).

[6] N.G. van Kampen, Stochastic Processes in Physics and Chemistry (North-Holland, Amsterdam, 1981).

[7] C.W. Gardiner, Quantum Noise (Springer, Berlin, 1991).

[8] U. Weiss, Quantum Dissipative Systems (Wold Scientific, Singapore, 1999).

[9] D. Zubarev, V. Morozov, and G. Röpke, Statistical mechanics of nonequilibrium processes, Vol. 2 (Academie Verlag, Berlin, 1997) p. 52.

[10] H.J. Carmichael, An open system approach to quantum optics (Springer, Berlin. 1993). 
[11] Yu.L. Klimontovich, Statistical theory of open systems (Kluwer Academic Publishers, Dordrecht, 1995).

[12] A.O. Caldeira and A.J. Leggett, Phys. Rev. Lett. 46, 211 (1981); Phys. Rev. Lett. 48, 1571 (1982); Ann. Phys. (N.Y.) 149, 374 (1983).

[13] V. V. Dodonov and V. I. Man'ko, Density Matrices and Wigner Functions of Quasiclassical Quantum Systems, Proc. Lebedev Phys. Inst. of Sciences, Vol. 167, ed. A. A. Komar (Nova Science, Commack, N. Y., 1987).

[14] K. Lindenberg and B. J. West, The Nonequilibrium Statistical Mechanics of Open and Closed Systems (VCH Publishers, Inc., New York, 1990); K. Lindenberg and B. J. West, Phys. Rev. A 30, 568 (1984).

[15] A. Isar, A. Sandulescu, H. Scutaru, E. Stefanescu, and W. Scheid, Int. J. Mod. Phys. E 3, 635 (1994).

[16] H.P. Breuer, F. Petruccione, The Theory of Open Quantum Systems (Oxford University Press, Oxford, 2002).

[17] G.W. Ford, J.T. Lewis, R.F. O’Connell, Phys. Rev. A 36, 1466 (1987); A 37, 4419 (1988).

[18] G.Y. Hu and R.F. O'Connell, Physica A 151, 33 (1988); Phys. Rev. B 36, 5798 (1987).

[19] X.L. Li, G.W. Ford, R.F. O’Connell, Phys. Rev. A 41, 5287 (1990); ibid 42, 4519 (1990); Physica A 193, 575 (1993).

[20] X.L. Li, G.W. Ford, R.F. O’Connell, Phys. Rev. E 53, 3359 (1996).

[21] S. Dattagupta and J. Singh, Phys. Rev. Lett. 79, 961 (1997).

[22] Th.M. Nieuwenhuizen and A.E. Allahverdyan, Phys. Rev. E 66, 036102 (2002).

[23] Z. Kanokov, Yu.V. Palchikov, G.G. Adamian, N.V. Antonenko, and W. Scheid, Phys. Rev. E 71, 016121 (2005); Sh.A. Kalandarov, Z. Kanokov, G.G. Adamian, and N.V. Antonenko, Phys. Rev. E 75, 0311115 (2007).

[24] G.G. Adamian, N.V. Antonenko, Z. Kanokov, and V.V. Sargsyan, Theor. Math. Phys. 145, $1443(2005)$.

[25] V.V. Sargsyan, Z. Kanokov, G.G. Adamian, and N.V. Antonenko, Phys. Rev. C 77, 024607 (2008).

[26] V.V. Sargsyan, Z. Kanokov, G.G. Adamian, and N.V. Antonenko, Phys. Part. Nuclei 41, 175 (2010).

[27] R.A. Kuzyakin, V.V. Sargsyan, G.G. Adamian, and N.V. Antonenko, Phys.Rev.A 83, 062117 
(2011); A 84, 032117 (2011).

[28] K. Wen, F. Sakata, Z.-X. Li, X.-Z. Wu, Y.-X. Zhang, and S.-G. Zhou, Phys. Rev. Lett. 111, 012501 (2013).

[29] D. Lacroix, V.V. Sargsyan, G.G. Adamian, and N.V. Antonenko, Eur. Phys. J. B 88, 89 (2015).

[30] V.V. Sargsyan, D. Lacroix, G.G. Adamian, and N.V. Antonenko, Phys. Rev. A 95, 032119 (2017).

[31] V.V. Sargsyan, D. Lacroix, G.G. Adamian, and N.V. Antonenko, Phys. Rev. A 90, 022123 (2014).

[32] V.V. Sargsyan, D. Lacroix, G.G. Adamian, and N.V. Antonenko, Phys. Rev. A 96, 012114 (2017).

[33] H.W. Lewis, Phys. Rev. 92, 1149 (1953); 100, 641 (1955).

[34] E.S. Borovik, Doklady Acad. Nauk S.S.S.R. 70, 601 (1950).

TABLE I: Experimental (asterisks) 34] and theoretical characteristics of Zn at two temperatures.

\begin{tabular}{ccccc}
\hline $\begin{array}{c}\text { Temperature* } \\
T(\mathrm{~K})\end{array}$ & $\begin{array}{c}\text { Resistance }^{*}, \rho_{x x}, \\
\times 10^{-11}(\mathrm{Ohm} \cdot \mathrm{m})\end{array}$ & $\begin{array}{c}\text { Mobility, } \\
\mu\left(\mathrm{m}^{2} / \mathrm{V} \cdot \mathrm{s}\right)\end{array}$ & $\begin{array}{c}\Gamma=B_{\text {max }}^{*}, \\
(\text { Tesla })\end{array}$ & $\begin{array}{c}\text { Max. Hall } \\
\text { angle }^{*}, \Theta_{H}\end{array}$ \\
\hline 4.22 & 2.6555 & 50.25 & 0.37 & $1.6^{\circ}$ \\
20.4 & 35.595 & 1.1 & 1.35 & $9.37^{\circ}$ \\
\hline
\end{tabular}



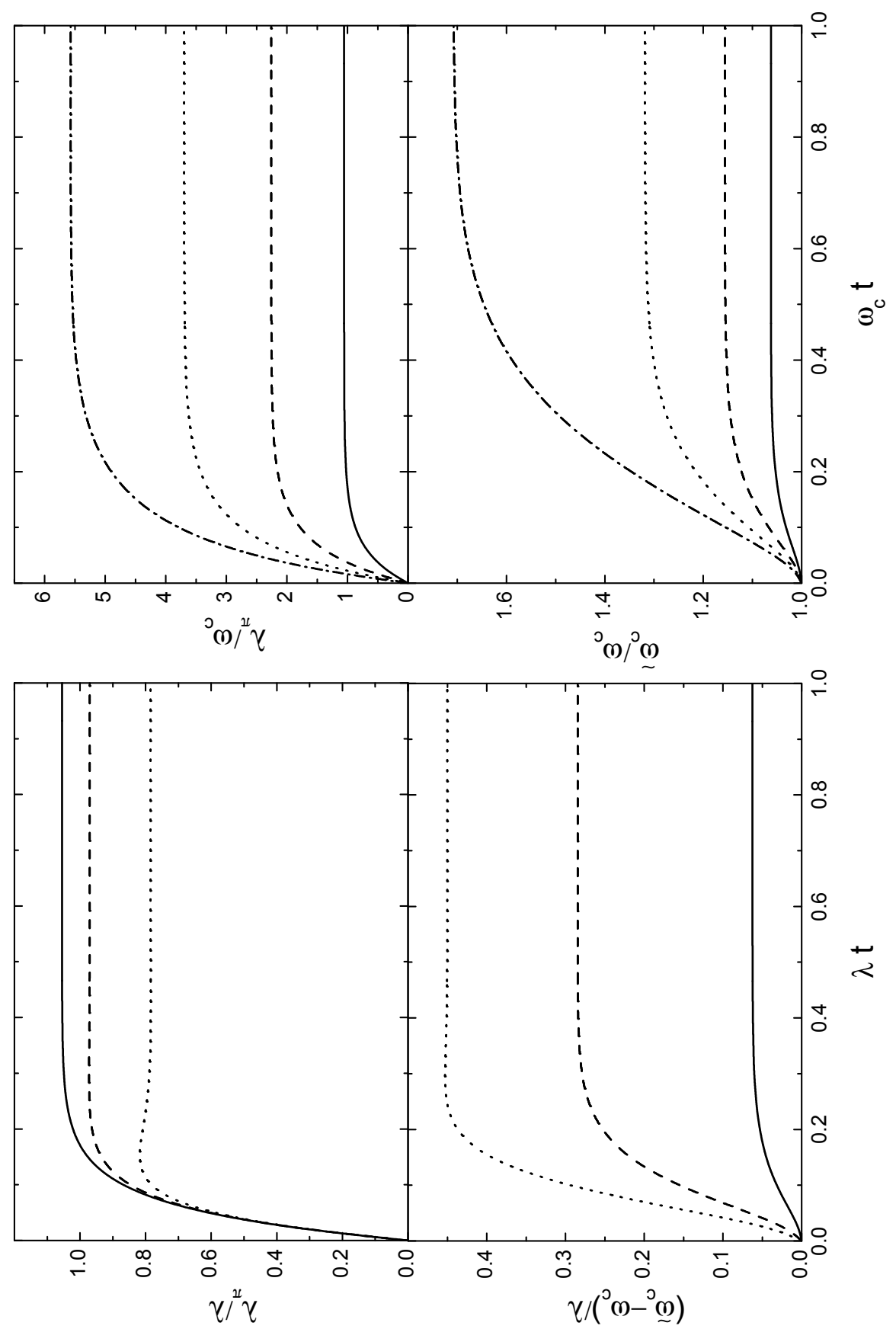

FIG. 1: The calculated friction coefficient and cyclotron frequency as functions of time. The results for the frequencies $\frac{\omega_{c}}{\lambda}=1,5$, and 10 of the external magnetic field at the fixed Markovian friction coefficient $\lambda$ are presented by solid, dashed, and dotted lines, respectively (left side). The results for the Markovian friction coefficient $\left(\lambda=\lambda_{x}=\lambda_{y}^{18}\right) \frac{\lambda}{\omega_{c}}=1,2,3$, and 4 at the fixed external magnetic field $\omega_{r}$ are presented by solid. dashed. dotted. and dash-dotted lines. respectivelv (right side). 

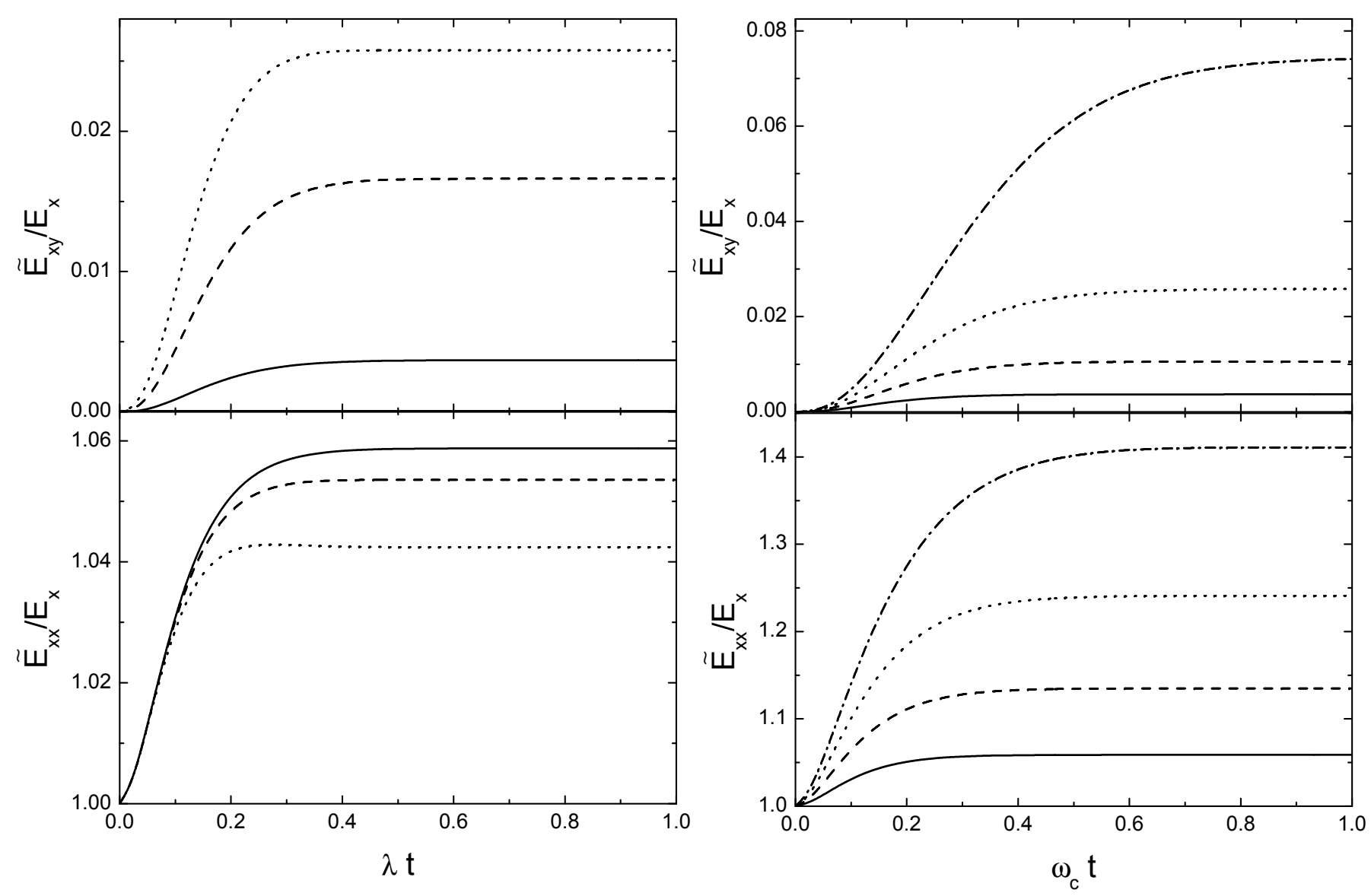

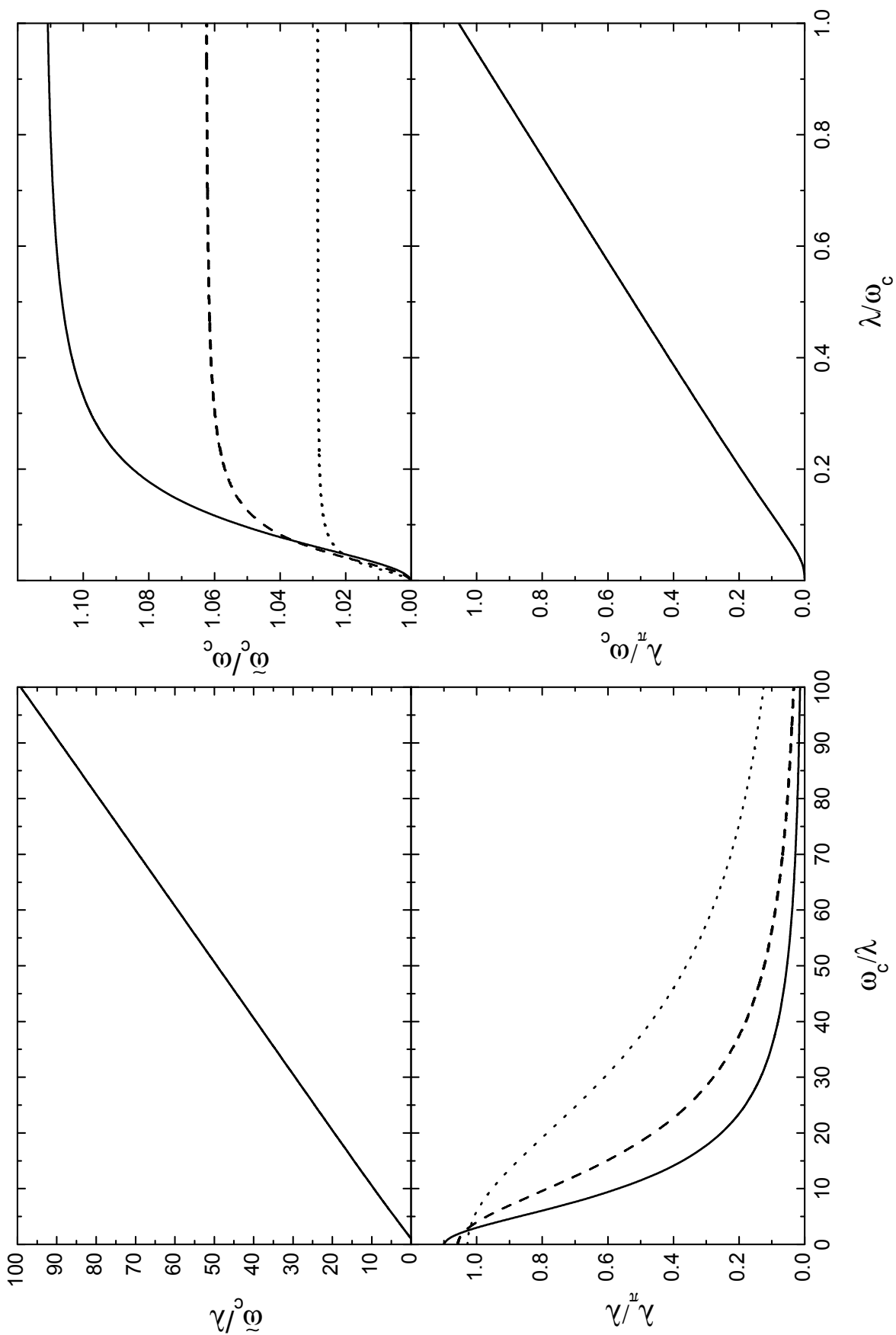

FIG. 3: The calculated asymptotic cyclotron frequencies and friction coefficients as functions of $\omega_{c}$ (left side) and $\lambda$ (right side). The solid, dashed, and dotted lines correspond to the calculations with $\gamma / \lambda=12,19$, and 38 , respectively. 


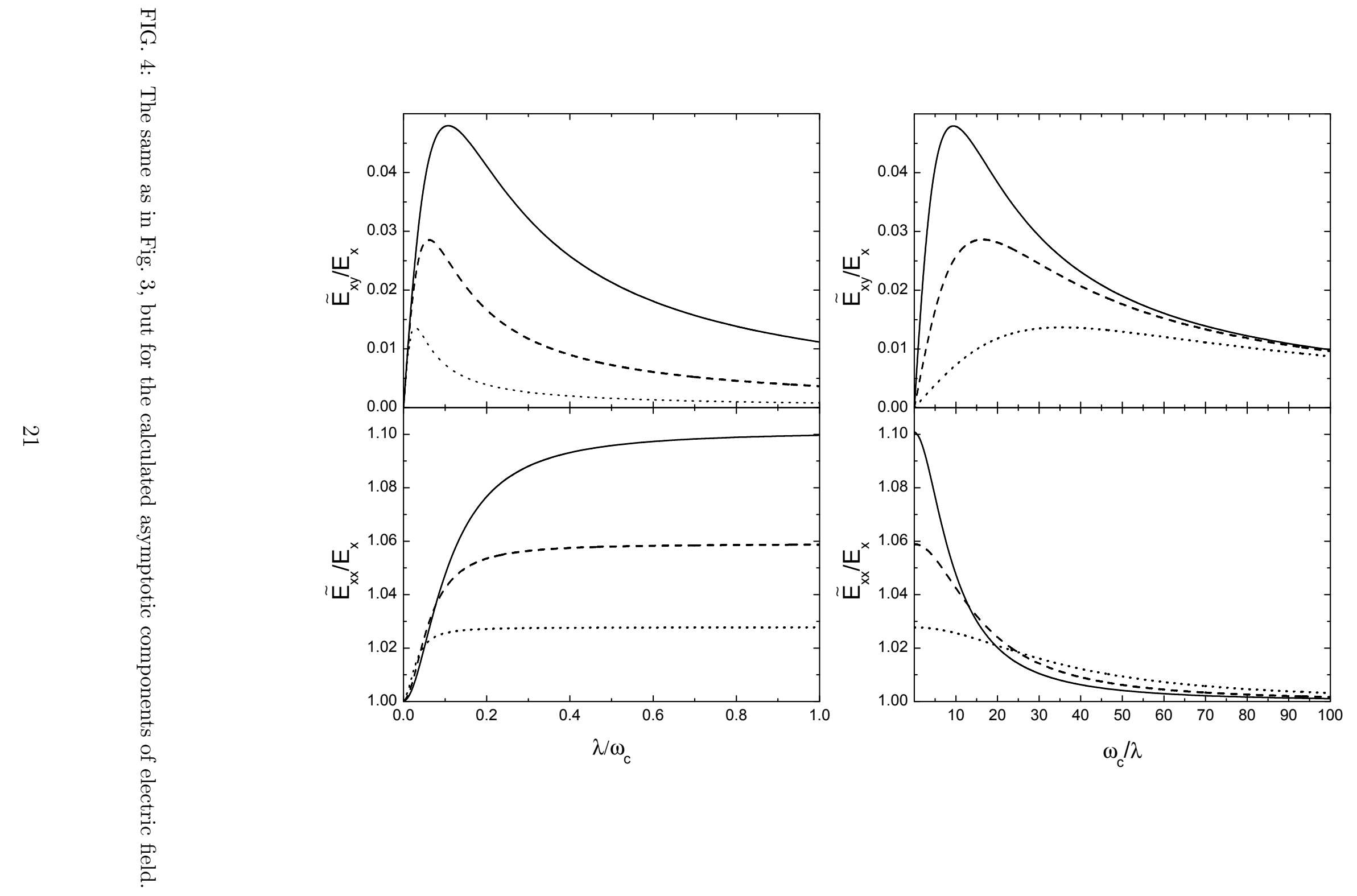




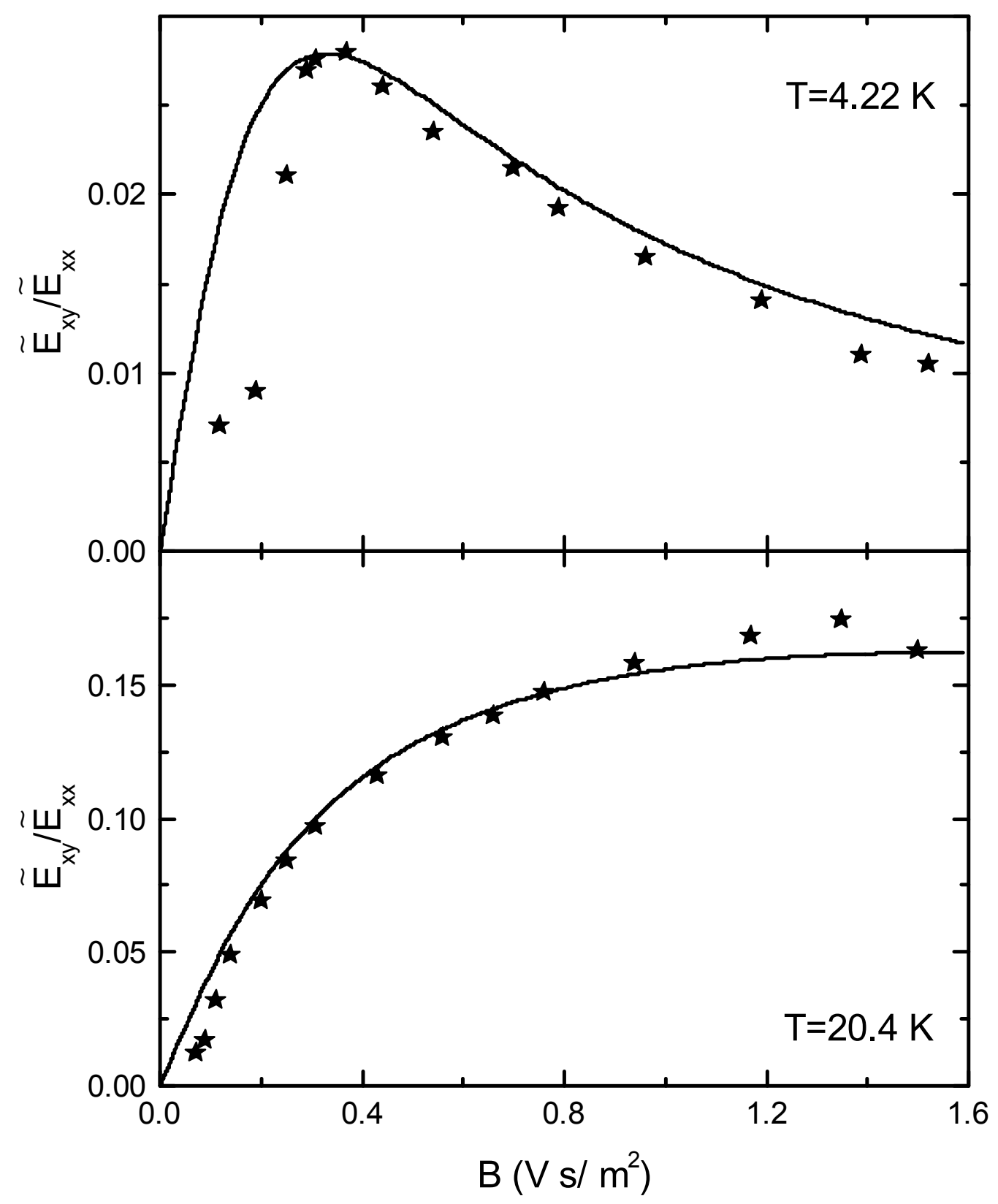

FIG. 5: The experimental [34] (symbols) and theoretical dependencies (lines) of the tangent of the Hall angle on magnetic field, $B$, for zinc at the temperatures $T$ indicated. 